TITLE:

\title{
Electron wave packet modeling of chemical bonding: Floating and breathing minimal packets with perfect-pairing valence-bond spin coupling
}

$\operatorname{AUTHOR}(S)$ :

Ando, Koji

\section{CITATION:}

Ando, Koji. Electron wave packet modeling of chemical bonding: Floating and breathing minimal packets with perfect-pairing valence-bond spin coupling. Chemical Physics Letters 2012, 523: 134-138

ISSUE DATE:

2012-01

URL:

http://hdl.handle.net/2433/153410

\section{RIGHT:}

(C) 2011 Elsevier B.V.; This is not the published version. Please cite only the published version.; この論文は出版社版でありません。引用の際に は出版社版をご確認ご利用ください。 


\title{
Electron wave packet modeling of chemical bonding: Floating and breathing minimal packets with perfect-pairing valence-bond spin coupling
}

\author{
Koji Ando* \\ Department of Chemistry, Graduate School of Science, Kyoto University, Sakyo-ku, Kyoto 606-8502, Japan
}

\begin{abstract}
A simple wave packet (WP) modeling of electrons in chemical bonding is examined. It is found that floating and breathing minimal Gaussian WPs with fully non-orthogonal perfect-pairing valence-bond spin coupling yield the ground state potential energy surfaces of $\mathrm{LiH}, \mathrm{BeH}_{2}, \mathrm{CH}_{2}$, and $\mathrm{H}_{2} \mathrm{O}$ molecules of comparable quality to a high-level ab initio electron-correlated calculations. A simple form of core pseudo-potential with two parameters is shown to give proper modeling of core-valence interactions.
\end{abstract}

\section{INTRODUCTION}

This Letter demonstrates that a simple wave packet (WP) modeling of electrons can yield reasonably accurate molecular potential energy surfaces. To each electron is ascribed a single squeezed coherent-state $\mathrm{WP}^{1}$, or a "minimal floating and breathing" Gaussian WP, with the antisymmetry of many-fermion wave function treated by the non-orthogonal valence-bond (VB) theory ${ }^{2,3}$. This is an extension of our previous studies on nuclear WP modeling of hydrogen-bond and proton transfer ${ }^{4-8}$, in which the time-dependent Hartree ansatz was deployed. Integration with the present study is thus anticipated to open a way to describe electron and nuclear quantum dynamics in a compact manner beyond the BornOppenheimer approximation ${ }^{9-14}$.

The importance of orbital breathing, i.e., variable width, was examined on $\mathrm{H}_{2}$ molecule immediately after the HeitlerLondon theory ${ }^{15}$. In the modern ab initio VB framework, the Breathing Orbital VB method was developed ${ }^{16}$. On the other hand, the orbital floating, i.e., displacement from the nuclear center, was examined on $\mathrm{H}_{2}$ molecule and concluded to be minor $^{17,18}$. For polyatomic molecules, the Floating Spherical Gaussian Orbital method was developed in the restricted Hartree-Fock framework ${ }^{19}$. Many other studies on floating orbitals have also been based on the molecular orbital (MO) scheme ${ }^{20-24}$.

It appears that the orbital floating in VB has not attracted much attention. This seems partly because of the historical prevalence of MO over VB. Another reason could be the sufficient flexibility attained by modern ab initio VB methods represented by the Spin Coupled VB ${ }^{25}$. It has been shown that the "semi-localized" or "overlap enhanced" orbitals almost eliminates the need for polar ionic VB structures ${ }^{25,26}$. Similarly, proper deforming of orbitals may diminish the need for floating them.

Nonetheless, our motivation to revisit the orbital floating in VB is to exploit it for dynamic WP simulations. The localized WP description fits better with VB than with MO. Moreover, the use of single (minimal) Gaussian WP greatly simplifies the equations of motion, whose conceptual and prac-

\footnotetext{
*Phone/Fax: +81-75-753-4018, E-mail: ando@kuchem.kyoto-u.ac.jp
}

tical assets have been illustrated by both analytical theories and molecular dynamics (MD) simulations for proton transfer and hydrogen-bonding systems ${ }^{4-8}$. We are thus motivated to explore an analogous WP modeling of electrons. As will be shown below, the results are encouraging, and so will be the extension toward combined electron-nuclear description.

There exist related studies on electron WPs. In nuclear and plasma physics, various fermion MD methods have been developed $^{27}$, some of which account for the antisymmetry via the "Pauli potential" that represents the triplet coupling of electron pairs. Recently, this method has been applied to ionization and dissociation dynamics of $\mathrm{D}_{3}^{+}$molecule under intense laser pulse ${ }^{28}$. Similar idea has been applied to a variety of systems by the electron Force-Field method ${ }^{29}$, in which an empirical mixture of singlet and triplet pair potentials has been parameterized. The present work differs from these in the treatment of antisymmetry and spin coupling.

The next section summarizes the theory and computation. Numerical results are discussed in Sec 3. The final section concludes.

A related preceding work has been published recently ${ }^{30}$. Novelties in this Letter include: (1) applications to $\mathrm{CH}_{2}$ and $\mathrm{H}_{2} \mathrm{O}$ that conventionally involve p-type atomic orbitals, (2) improved core pseudo-potential (CPP), and (3) assessment of frozen-core approximation.

\section{THEORY AND COMPUTATION}

Since the basic framework has been described in the preceding work ${ }^{30}$, a brief outline is given in a form specific to the present calculations.

\section{A. VB function}

We employ a VB function for $N$-electrons ${ }^{2}$,

$$
\Psi(1, \cdots, N)=\mathcal{A}\left[\Omega\left(\boldsymbol{q}_{1}, \cdots, \boldsymbol{q}_{N}\right) \Theta(1, \cdots, N)\right],
$$

in which $\Omega$ and $\Theta$ are the spatial and spin functions to be specified below. $\mathcal{A}$ is an antisymmetrizer. As used in ordinary VB methods, the spatial part $\Omega$ is described by a direct product of one-particle orbitals $\phi$,

$$
\Omega\left(\boldsymbol{q}_{1}, \cdots, \boldsymbol{q}_{N}\right)=\phi\left(\boldsymbol{q}_{1}\right) \cdots \phi\left(\boldsymbol{q}_{N}\right) .
$$


The key element in our modeling is the orbitals $\phi\left(\boldsymbol{q}_{i}\right)$ taken to be the squeezed coherent-state WPs characterized by the center and width coordinates and their conjugate momenta. These parameters follow equations of motion derived from the timedependent variational principle ${ }^{31}$. Presently, however, we restrict the target to the time-independent problem, so that the momentum parameters are nullified and the orbitals take the form

$$
\phi\left(\boldsymbol{q}_{i}\right)=\left(2 \pi \rho_{i}^{2}\right)^{-3 / 4} \exp \left(-\left|\boldsymbol{q}_{i}-\boldsymbol{r}_{i}\right|^{2} / 4 \rho_{i}^{2}\right),
$$

in which $\boldsymbol{r}_{i}$ and $\rho_{i}$ represent the WP center and width. They are variables independent of the nuclear coordinates; that is, the orbitals $\phi$ are "floating and breathing", in contrast with the ordinary VB (and MO) calculations.

In this study, the spin function $\Theta$ assumes the perfectpairing (PP) form ${ }^{2}$

$$
\Theta(1, \cdots, N)=\theta(1,2) \theta(3,4) \cdots \theta(N-1, N),
$$

in which $\theta$ is a pair spin function of singlet coupling

$$
\theta(1,2)=(\alpha(1) \beta(2)-\beta(1) \alpha(2)) / \sqrt{2} .
$$

Unlike the generalized $\mathrm{VB}^{32}$ and related methods that impose the "strong-orthogonality condition" 2 among different pairs, we fully take account of the non-orthogonality of orbitals $\phi$, by which the localized WP picture is maintained.

The energy $E=\langle\Psi|H| \Psi\rangle /\langle\Psi \mid \Psi\rangle$ of the VB function in Eq. (1) is calculated via the standard formula of the spin-free VB theory ${ }^{2}$, where

$$
\langle\Psi|H| \Psi\rangle=\sum_{P \in S_{N}} D_{11}^{[\lambda]}(P)\langle\Omega|H| P \Omega\rangle
$$

and

$$
\langle\Psi \mid \Psi\rangle=\sum_{P \in S_{N}} D_{11}^{[\lambda]}(P)\langle\Omega \mid P \Omega\rangle,
$$

in which $P$ is the permutation operator of the $N$-electron indices whose summation runs over all the $N$ ! permutations of the symmetric group $S_{N} . D_{11}^{[\lambda]}(P)$ is the first diagonal element of the irreducible representation matrix of $S_{N}$. The evaluation of $D_{11}^{[\lambda]}(P)$ for the PP case is particularly straightforward $^{33}$.

For the optimization of the WP center and width parameters, the method of Brent ${ }^{34}$ that does not require gradients is employed at the current implementation.

\section{B. Core pseudo-potential}

Since the fully non-orthogonal VB calculations suffer the so-called " $N$ !-problem" in the evaluation of Eqs (6) and (7), reduction of the number of active electrons greatly saves the computational cost. We therefore explore the use of a $\mathrm{CPP}^{35}$ of a form

$$
V_{\mathrm{cpp}}(r)=-\frac{Z-Z_{\mathrm{c}}}{r}\left(1-A_{\mathrm{c}} \exp \left(-r^{2} / 4 \rho_{\mathrm{c}}^{2}\right)\right),
$$

in which $A_{\mathrm{c}}$ and $\rho_{\mathrm{c}}$ are parameters specifying the amplitude and width of $V_{\text {cpp }}$, and $Z_{\mathrm{c}}$ is the number of core electrons. Previously ${ }^{30}$, we fixed it at $A_{\mathrm{c}}=1$ by which $V_{\text {cpp }}$ vanishes at $r=0$. This simplifies both the qualitative picture and the fitting procedure, though it is not an essential restriction. We treat in this work both $\rho_{\mathrm{c}}$ and $A_{\mathrm{c}}$ as parameters to be optimized. We set $Z_{\mathrm{c}}=2$ throughout this work.

\section{Frozen core approximation}

We also test freezing the parameters of 1s core orbitals. This leaves the $N$ !-problem intact, but reduces the cost for orbital optimizations. We first carry out calculations for three electron atoms, $\mathrm{Li}, \mathrm{Be}^{+}, \mathrm{C}^{3+}$, and $\mathrm{O}^{5+}$, whose results for $1 \mathrm{~s}$ electrons are then transferred frozen to the calculations of molecules.

\section{RESULTS AND DISCUSSION}

\section{A. Four electrons: LiH}

We first summarize in the next paragraph the previous ${ }^{30}$ results on $\mathrm{LiH}$ to be compared with the present ones with fully optimized CPP for the Li 1s electrons.

The potential energy curve from the all (four)-electron VB-PP calculation was of comparable quality to that from the multi-reference second-order Möller-Plesset theory ${ }^{36}$ with the correlation-consistent polarized valence double-zeta basis $\operatorname{set}^{37}$ (MRMP2/cc-pVDZ, by the program GAMESS ${ }^{38}$ ). Along the chemical bond formation, one of the two valence electron WPs moved from the Li nucleus position toward the bonding region, whereas the other WP remained near the $\mathrm{H}$ nucleus. The CPP with $A_{\mathrm{c}}=1$ gave a qualitatively correct dissociation curve, but underestimated the well-depth by $\sim 30$ $\%$. The optimized CPP width parameter was $\rho_{\mathrm{c}}=0.7 \mathrm{bohr}$.

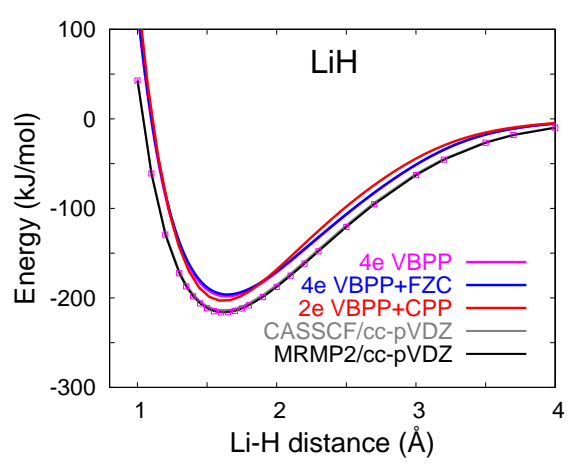

FIG. 1: Potential energy curves of $\mathrm{LiH}$, from full(four)-electron VB$\mathrm{PP}$, the same with frozen-core (FZC) approximation, two-electron VB-PP with core pseudo-potential (CPP), and CASSCF and MRMP2 references with cc-pVDZ basis set. 
Table 1

Equilibrium bond length $r_{\mathrm{e}}$, dissociation energy $D_{\mathrm{e}}$ (per bond), and vibrational frequency $\omega_{\mathrm{e}}$ (of symmetric stretch for $\mathrm{XH}_{2}$ molecules).

\begin{tabular}{|c|c|c|c|}
\hline & $r_{\mathrm{e}}(\AA)$ & $D_{\mathrm{e}}(\mathrm{kJ} / \mathrm{mol})$ & $\omega_{\mathrm{e}}\left(\mathrm{cm}^{-1}\right)$ \\
\hline \multicolumn{4}{|l|}{$\mathrm{LiH}$} \\
\hline $4 \mathrm{eVB}$ & 1.64 & 198 & 1430 \\
\hline $2 \mathrm{eVB}+\mathrm{CPP}$ & 1.62 & 203 & 1580 \\
\hline CASSCF $^{a}$ & 1.62 & 214 & 1380 \\
\hline MRMP $2^{a}$ & 1.62 & 216 & 1380 \\
\hline other works ${ }^{b}$ & 1.595 & 242.7 & 1406.2 \\
\hline \multicolumn{4}{|l|}{$\mathrm{BeH}_{2}$} \\
\hline $6 \mathrm{eVB}$ & 1.32 & 295 & 2330 \\
\hline $4 \mathrm{eVB}+\mathrm{CPP}$ & 1.33 & 296 & 2440 \\
\hline CASSCF & 1.36 & 243 & 1990 \\
\hline MRMP2 & 1.34 & 280 & 2060 \\
\hline other works ${ }^{\mathrm{c}}$ & 1.326 & 309.5 & 2178.9 \\
\hline \multicolumn{4}{|l|}{$\mathrm{CH}_{2}$} \\
\hline $8 \mathrm{eVB}$ & 1.15 & 322 & 2550 \\
\hline $6 \mathrm{eVB}+\mathrm{CPP}$ & 1.24 & 282 & 2280 \\
\hline CASSCF & 1.14 & 317 & 2730 \\
\hline MRMP2 & 1.13 & 336 & 2740 \\
\hline other works ${ }^{d}$ & 1.107 & 379.2 & 2846 \\
\hline \multicolumn{4}{|l|}{$\mathrm{H}_{2} \mathrm{O}$} \\
\hline $10 \mathrm{eVB}+\mathrm{FZC}$ & 1.05 & 265 & 3340 \\
\hline $8 \mathrm{eVB}+\mathrm{CPP}$ & 1.03 & 277 & 3550 \\
\hline CASSCF & 0.97 & 385 & 3650 \\
\hline MRMP2 & 0.97 & 431 & 3708 \\
\hline other works ${ }^{\mathrm{e}}$ & 0.958 & 487.0 & 3567 \\
\hline
\end{tabular}

In this work, both $\rho_{\mathrm{c}}$ and $A_{\mathrm{c}}$ have been optimized toward the target reference of the full (four-electron) VB-PP curve. The result is displayed in Figure 1 together with the full VB$\mathrm{PP}$ and the CASSCF and MRMP2/cc-pVDZ. The equilibrium bond length $r_{\mathrm{e}}$, dissociation energy $D_{\mathrm{e}}$, and vibrational fre-

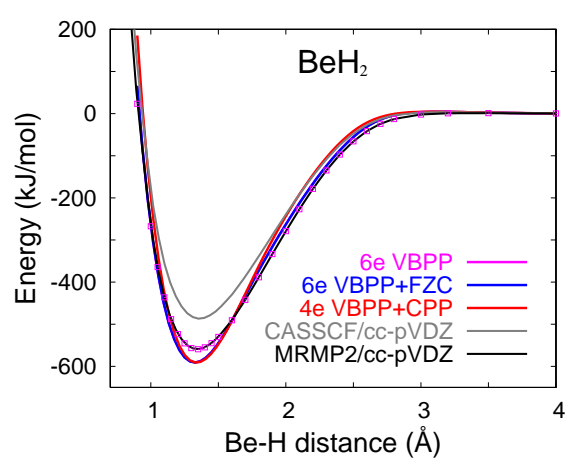

FIG. 2: Potential energy curves of a linear molecule $\mathrm{BeH}_{2}$ along the symmetric Be-H stretch. The legend is similar to that in Figure 1. The curves from VB-PP and VB-PP+FZC are indistinguishable such that the former is overdrawn by the latter. quency $\omega_{\mathrm{e}}$ are listed in Table 1 . With respect to the MRMP2 reference, the full VB-PP overestimate $r_{\mathrm{e}}$ and $\omega_{\mathrm{e}}$ by $1 \%$ and $4 \%$ and underestimate $D_{\mathrm{e}}$ by $8 \%$. The CASSCF and MRMP2 curves overlap well with a difference of $2 \mathrm{~kJ} / \mathrm{mol}$ in $D_{\mathrm{e}}$, indicating that the dynamic correlation effect in the bonding is small, as expected in this molecule. The frozen-core approximation gave the results almost indistinguishable from the full-electron calculation; this applies to all the other three molecules, $\mathrm{BeH}_{2}, \mathrm{CH}_{2}$, and $\mathrm{H}_{2} \mathrm{O}$, and thus will not be repeated in the following sections. The agreement of the CPP curve with the full VB-PP indicate that the core-valence interaction is properly described by the simple form of $V_{\mathrm{cpp}}$ in Eq. (8). The optimized parameters are $\rho_{\mathrm{c}}=0.29 \mathrm{bohr}$ and $A_{\mathrm{c}}=7.5$.

\section{B. Six electrons: $\mathrm{BeH}_{2}$}

We next examine a six-electron molecule $\mathrm{BeH}_{2}$. This was studied previously with four-electron VB-PP with a CPP for the Be 1 s electrons ${ }^{30}$, in which the CPP with $A_{\mathrm{c}}=1$ underestimated the potential well-depth along the symmetric stretch coordinate similarly to the $\mathrm{LiH}$ case. Here we present new results with all (six)-electron VB-PP and the CPP with both $\rho_{\mathrm{c}}$ and $A_{\mathrm{c}}$ optimized.

The results are shown in Figure 2 and Table 1. The accuracy toward the MRMP2 reference again appears reasonably high. The dynamic correlation energy measured by the difference between CASSCF and MRMP2 amounts to $13 \%$ of $D_{\mathrm{e}}$ of MRMP2. In this regard, the full VB-PP overshoots it by 41 $\%$. However, $D_{\mathrm{e}}$ by full VB-PP still underestimates the experimental value by $15 \mathrm{~kJ} / \mathrm{mol}$. If we consider that the present VB-PP is unlikely to better describe the dynamic correlation than MRMP2, the overestimate of $D_{\mathrm{e}}$ may be attributed to a poorer description of the correlation in the Be atom in the dissociation limit than in the bonding region.

The CPP parameters for Be 1s electrons, fitted toward the full VB-PP reference, are $\rho_{\mathrm{c}}=0.14 \mathrm{bohr}$ and $A_{\mathrm{c}}=12.5$.

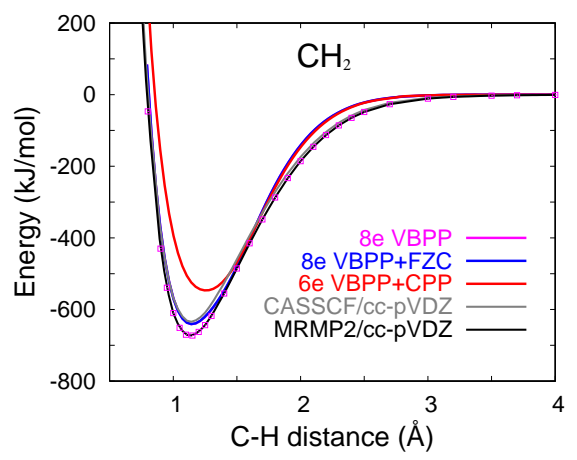

FIG. 3: Potential energy curve of $\mathrm{CH}_{2}$ along the symmetric C$\mathrm{H}$ stretch. The $\mathrm{HCH}$ angle is fixed at $99.9^{\circ}$, as optimized by CASSCF/cc-pVDZ. The legend is similar to that in Figure 1. The curves from VB-PP and VB-PP+FZC are indistinguishable such that the former is overdrawn by the latter. 


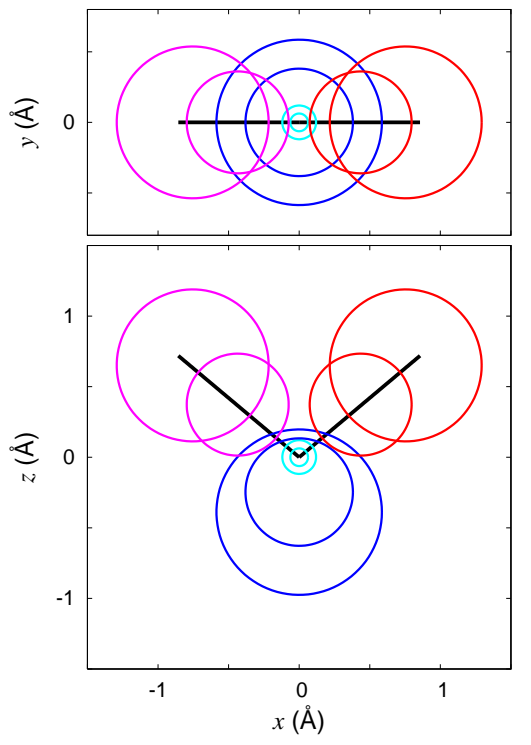

FIG. 4: Optimized electron wave packets for $\mathrm{CH}_{2}$ from the full VB$\mathrm{PP}$ calculation. Each wave packet is represented by a circle of radius $\rho_{\mathrm{c}}$. Paired electrons are in the same color. The thick black lines represent the interatomic bond.

Again, the simple form of Eq. (8) yields accurate description of the core-valence interaction within the framework.

\section{Eight electrons: $\mathrm{CH}_{2}$}

The next illustration is an eight-electron molecule $\mathrm{CH}_{2}$. This poses a new test for the description of electronic structures that conventionally involve p-type atomic orbitals.

The results are shown in Figure 3. In this case, the full VB-PP curve appears rather closer to the CASSCF reference; Table 1 indicates that the full VB-PP yields $26 \%$ of the dynamic correlation energy of the CASSCF-MRMP2 reference. Table 1 also shows that $r_{\mathrm{e}}$ is overestimated by $2 \%$ and $\omega_{\mathrm{e}}$ is underestimated by $7 \%$ from the MRMP 2 reference.

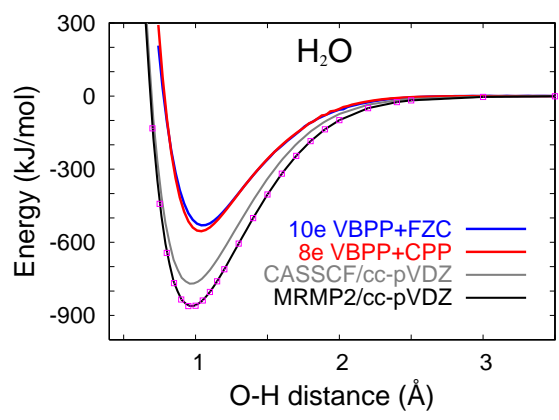

FIG. 5: Potential energy curve of $\mathrm{H}_{2} \mathrm{O}$ along the symmetric O$\mathrm{H}$ stretch. The $\mathrm{HOH}$ angle is fixed at $101.2^{\circ}$, as optimized by CASSCF/cc-pVDZ. The legend is similar to that in Figure 1.

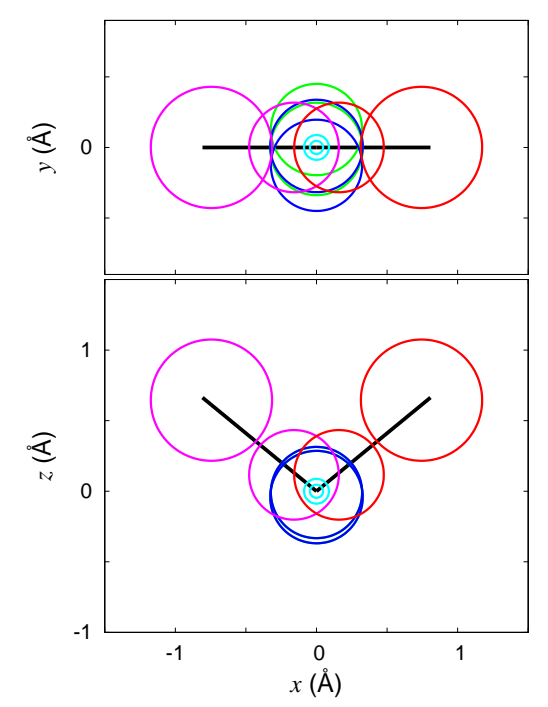

FIG. 6: The same as Figure 4 but for $\mathrm{H}_{2} \mathrm{O}$.

With the CPP for $\mathrm{C} 1 \mathrm{~s}$ electrons, the best fit shown in the figure was obtained with $\rho_{\mathrm{c}}=0.066 \mathrm{bohr}$ and $A_{\mathrm{c}}=16.0$. As seen, the fitting was not so successful as the $\mathrm{LiH}$ and $\mathrm{BeH}_{2}$ cases. This might be due to a difficulty in describing both core-bonding and core-lone-pair interactions by the simple form of CPP in Eq. (8). Further study will be needed on this issue, which will be based on a detailed analysis of the WPs as described next.

The electron WPs in $\mathrm{CH}_{2}$ are displayed in Figure 4, each represented by a circle of radius $\rho_{\mathrm{c}}$. The core, lone-pair, and covalent-bond electrons are clearly seen. All the WPs have centers on the molecular plane. The core electron WPs are localized at the $\mathrm{C}$ nuclear position with small widths. The lone-pair WPs extend toward the direction opposite from the $\mathrm{CH}$ bonds. Each covalent $\mathrm{CH}$ bond is described by a WP pair; one shrinks in the bonding region and the other expands around the $\mathrm{H}$ nucleus. These show an intriguing contrast with the $\mathrm{H}_{2} \mathrm{O}$ case to be discussed in Section III D. Toward the dissociation limit, the WPs around the $\mathrm{H}$ atoms come closer to the nuclei while the remaining WPs are localized around the C nucleus.

The success of the present full VB-PP without the conventional p-type orbitals is not so surprising as it might appear. As the density functional theory has clarified, the ground state electronic energy is determined by the electron density. The ground state total electronic wave functions are normally nodeless, in which the primary roles of the nodal basis functions or one-electron orbitals are to introduce density polarizations. The argument applies both in the bonding region and the dissociation limit. In this regard, the description of electronic excited states will pose a severer challenge to the present method, which is currently under study in connection with the time-dependent response theory, but shall be reserved for future reports. 


\section{Ten electrons: $\mathrm{H}_{2} \mathrm{O}$}

We finally examine a 10-electron molecule $\mathrm{H}_{2} \mathrm{O}$. This was our primary motivation, as we have recently implemented a semiquantum nuclear WP simulation of liquid water with a classical force-field potential ${ }^{7,8}$ : Combination with the electron WPs is thus awaited.

Figure 5 shows the resultant potential energy curves. In contrast with the other cases studied so far, the full-electron VB-PP notably underestimate the binding energy. This might be due to an insufficient description of the dynamic correlation effect involving the lone-pair electrons that are contracted around the $\mathrm{O}$ atom, as discussed below with the plots in Figure 6. This issue will require further analysis, and we will return to it with ideas for improvements in the concluding section.

The potential curve with $\mathrm{CPP}$ for $\mathrm{O}$ 1s electrons is included in Figure 5. To reduce the computational cost for determining the CPP parameters, we extrapolated from the results of $\mathrm{LiH}$, $\mathrm{BeH}_{2}$, and $\mathrm{CH}_{2}$. That is, we first observed that both $\rho_{\mathrm{c}}$ and $A_{\mathrm{c}}$ depended almost linearly on $1 /\left(Z-Z_{\mathrm{c}}\right)$. We thus carried out a least-squares fitting and determined the parameters for $\mathrm{O} 1 \mathrm{~s}$ CPP to be $\rho_{\mathrm{c}}=0.041 \mathrm{bohr}$ and $A_{\mathrm{c}}=17.2$. Figure 5 and Table 1 show that these CPP parameters reproduce the full VB-PP reasonably well. This also seems to imply that the modeling of core-valence interactions is not the primary cause for the underestimate of binding energy.

Figure 6 displays the electron WPs in $\mathrm{H}_{2} \mathrm{O}$. In comparison to the $\mathrm{CH}_{2}$ case in Figure 4, most of the WPs except for the two around the $\mathrm{H}$ nuclei are contracted and localized near the $\mathrm{O}$ nucleus. This is reasonable for the larger Coulomb attraction from the nuclear charge $Z=8$. The centers of two WP pairs that correspond to the lone-pair electrons are dislocated above and below the molecular plane: in one of the pairs, the shifts are $+0.13 \AA$ and $-0.01 \AA$.

\section{CONCLUDING REMARKS}

We have discussed the potential utility of a simple WP modeling of electrons, floating and breathing minimal Gaussian WPs with VB-PP coupling, for describing molecular poten- tial energy surfaces. The results are encouraging, indicating that the correlation and polarization effects are properly taken into account; in a simple assignment, the static correlation is described by the VB form, the dynamic correlation by the WP breathing, and the polarization by the WP floating.

Nevertheless, needs for improvements are apparent. Firstly, the underestimate of the binding energy in the particular case of $\mathrm{H}_{2} \mathrm{O}$ should be clarified. Secondly, the inclusion of dynamic correlation effect is not always quantitative. Thirdly, we have observed a tendency to overestimate the forceconstants along the bending angles: for example, the full VBPP calculation of $\mathrm{CH}_{2}$ overestimated the $\mathrm{HCH}$ bending vibrational frequency by a factor of 1.49 and underestimated the equilibrium $\mathrm{HCH}$ angle by $4^{\circ}$ compared to the MRMP 2 reference. These issues may be attributed to the use of spherical WPs, and could then be remedied by introducing more flexible WPs such as ellipsoids. Indeed, we have recently implemented ellipsoidal nuclear WPs into MD simulation of water with a classical force-field potential ${ }^{49}$. The extension to electron WPs is now under development.

The computational efficiency should also be improved. Our current computer code employs an optimization method that does not require gradients ${ }^{34}$, but the use of gradients will enhance the efficiency and robustness. Moreover, the present study has left the $N$ !-problem intact, and the nonorthogonality of WPs has fully been taken into account at the cost of computing all the $N$ ! overlap and Hamiltonian matrix elements. In order to achieve large-scale simulations, however, it is essential to seek for efficient approaches to dealing with many-body intermolecular potentials. To this end, decoupling approximations into subgroups of electrons by exploiting the localized nature of non-orthogonal WPs will be of potential use. Works along these lines will be reported in due course.

\section{Acknowledgment}

The author acknowledges support from KAKENHI Nos. 20108017 (" $\pi$-space") and 22550012.

1 J.R. Klauder, B.S. Skagerstam (Eds.), Coherent States: Applications in Physics and Mathematical Physics, World Scientific, Singapore, 1985.

2 R. McWeeny, Methods of Molecular Quantum Mechanics, Academic, London, 1992.

${ }^{3}$ G.A. Gallup, Valence Bond Methods: Theory and applications, Cambridge University Press, Cambridge, 2002.

${ }^{4}$ K. Ando, J. Chem. Phys. 121 (2004) 7136.

K. Ando, J. Chem. Phys. 125 (2006) 014104.

${ }^{6}$ N. Sakumichi, K. Ando, J. Chem. Phys. 128 (2008) 164516.

${ }^{7}$ K. Hyeon-Deuk, K. Ando, J. Chem. Phys. 131 (2009) 064501.

${ }^{8}$ K. Hyeon-Deuk, K. Ando, J. Chem. Phys. 132 (2010) 164507.

9 K. Takatsuka, Int. J. Quant. Chem. 109 (2009) 2131.

10 E. Deumens, Y. Öhrn, J. Phys. Chem. A 105 (2001) 2660.
${ }^{11}$ M. Cafiero, S. Bubin, L. Adamowicz, Phys. Chem. Chem. Phys. 5 (2003) 1491.

12 T. Ishimoto, M. Tachikawa, U. Nagashima, Int. J. Quant. Chem. 109 (2009) 2677.

${ }^{13}$ C. Ko, M.V. Pak, C. Swalina, S. Hammes-Schiffer, J. Chem. Phys. 135 (2011) 054106.

${ }^{14}$ M. Hoshino, H. Nishizawa, H. Nakai, J. Chem. Phys. 135 (2011) 024111.

15 S.C. Wang, Phys. Rev. 31 (1928) 579.

${ }^{16}$ P.C. Hiberty, S. Humbel, C.P. Byrman, J.H. van Lenthe, J. Chem. Phys. 101 (1994) 5869.

${ }^{17}$ H. Shull, D.D. Ebbing, J. Chem. Phys. 28 (1958) 866

18 C.M. Reeves, J. Chem. Phys. 39 (1963) 1.

19 A.A. Frost, in Modern Theoretical Chemistry, Vol. 3, Plenum, 
New York, 1977.

${ }^{20}$ H. Nakatsuji, S. Kanayama, S. Harada, T. Yonezawa, J. Am. Chem. Soc. 100 (1978) 7528.

${ }^{21}$ H. Huber, J. Mol. Struct. (Theochem) 76 (1981) 277.

${ }^{22}$ T. Helgaker, J. Almlof, J. Chem. Phys. 89 (1988) 4889.

${ }^{23}$ K. Hirao, K. Mogi, J. Comp. Chem. 13 (1992) 457.

${ }^{24}$ M. Tachikawa, K. Taneda, K. Mori, Int. J. Quant. Chem. 75 (1999) 497.

25 J. Gerratt, D.L. Cooper, P.B. Karadakov, M. Raimondi, Chem. Soc. Rev. (1997) 87.

${ }^{26}$ R. McWeeny, Int. J. Quant. Chem. 34 (1988) 25.

${ }^{27}$ H. Feldmeier, J. Schnack, Rev. Mod. Phys. 72 (2000) 655.

${ }^{28}$ E. Löstedt, T. Kato, K. Yamanouchi, Phys. Rev. Lett. 106 (2011) 203001.

29 J.T. Su, W.A. Goddard III, J. Chem. Phys. 131 (2009) 244501.

${ }^{30}$ K. Ando, Bull. Chem. Soc. Jpn. 82 (2009) 975.

${ }^{31}$ P. Kramer, M. Saraceno, Geometry of the Time-Dependent Variational Principle in Quantum Mechanics, Springer, Berlin, 1981.

32 W.A. Goddard III, T.H. Dunning Jr., W.J. Hunt, P.J. Hay, Acc. Chem. Res. 6 (1973) 368.

33 J. Li, W. Wu, Theor. Chim. Acta 89 (1994) 105.

${ }^{34}$ R. Brent, Algorithms for Minimization without Derivatives, Dover, New York, 2002.

35 V. Bonifacic, S. Huzinaga, J. Chem. Phys. 60 (1974) 2779.

${ }^{36}$ K. Hirao, Chem. Phys. Lett. 190 (1992) 374.
37 T.H. Dunning Jr., J. Chem. Phys. 90 (1989) 1007.

${ }^{38}$ M.W. Schmidt, K.K. Baldridge, J.A. Boatz, S.T. Elbert, M.S. Gordon, J.H. Jensen, S. Koseki, N. Matsunaga, K.A. Nguyen, S. J. Su, T.L. Windus, M. Dupuis, J.A. Montgomery, J. Comp. Chem. 14 (1993) 1347

39 F.B. Orth, W.C. Stwalley, J. Mol. Spectrosc. 76 (1979) 17.

${ }^{40}$ W.C. Stwalley, W.T. Zemke, J. Phys. Chem. Ref. Data 22 (1993) 87.

41 A. Shayesteh, K. Tereszchuk, P.F. Bernath, R. Colin, J. Chem. Phys. 118 (2003) 3622.

42 J.M.L. Martin, Chem. Phys. Lett. 273 (1997) 98.

${ }^{43}$ H. Petek, D.J. Nesbitt, D.C. Darwin, P.R. Ogilby, C.B. Moore, D.A. Ramsay, J. Chem. Phys. 91 (1989) 6566.

${ }^{44}$ J.M.L. Martin, J.P. Francois, R. Gijbels, Chem. Phys. Lett. 163 (1989) 387.

45 G.V. Hartland, D. Qin, H. Dai, J. Chem. Phys. 98 (1993) 2469.

46 A.R. Hoy, P.R. Bunker, J. Mol. Spectrosc. 74 (1979) 1.

${ }^{47}$ B. Ruscic, A.F. Wagner, L.B. Harding, R.L. Asher, D. Feller, D.A. Dixon, K.A. Peterson, Y. Song, X. Qian, C.Y. Ng, J. Liu, W. Chen, D.W. Schwenke, J. Phys. Chem. A 106 (2002) 2727.

48 T. Shimanouchi, Talbes of Molecular Vibrational Frequencies Consolidated, National Bureau of Standards, 1972.

49 J. Ono, K. Ando, submitted for publication. 